\title{
Seeking massless dark photons in the decays of charmed hadrons
}

\author{
Jhih-Ying $\mathrm{Su}^{1}$ and Jusak Tandean $\odot^{1,2}$ \\ ${ }^{1}$ Department of Physics, National Taiwan University, Taipei 106, Taiwan \\ ${ }^{2}$ Physics Division, National Center for Theoretical Sciences, Hsinchu 300, Taiwan
}

(Received 29 June 2020; accepted 19 November 2020; published 23 December 2020)

\begin{abstract}
A massless dark photon could affect standard-model particles only via higher-dimensional operators and would therefore have eluded recent searches for its massive counterpart, which were based on the assumption that the latter had renormalizable interactions with known fermions due to gauge kinetic mixing. In this study, we entertain the possibility that the massless dark photon has non-negligible flavorchanging dipole-type couplings with the $u$ and $c$ quarks, giving rise to the decays of charmed hadrons into a lighter hadron plus missing energy carried away by the dark photon. We propose to investigate decays of this kind, especially those in which the parents are the charmed pseudoscalar mesons $D^{+}, D^{0}$, and $D_{s}^{+}$and singly charmed baryons $\Lambda_{c}^{+}, \Xi_{c}^{+}$, and $\Xi_{c}^{0}$. Employing a simplified new-physics model satisfying the relevant constraints, we find that the branching fractions of these processes could be as large as several times $10^{-5}$. This suggests that one or more of them might in the near future fall within reach of the ongoing Belle II and BESIII experiments. Since the same underlying operators are responsible for all of these transitions, detecting one of them automatically implies particular predictions for the others, allowing for additional experimental checks on the massless-dark-photon scenario.
\end{abstract}

DOI: 10.1103/PhysRevD.102.115029

\section{INTRODUCTION}

Attempts to address longstanding open questions in physics, such as the nature and origin of neutrino mass and the particle identity of cosmic dark matter, have increasingly postulated the existence of a dark sector beyond the standard model (SM). It is reasonable to expect that the new dark sector not only provides resolutions to some of those major puzzles but also furnishes extra ingredients which will facilitate further empirical access to it. Among the most attractive ones is a dark Abelian gauge group, $\mathrm{U}(1)_{D}$, under which all SM fields are singlets. This symmetry may be spontaneously broken or stay unbroken, causing the associated gauge boson, the dark photon, to gain mass or remain massless, respectively.

Whether it is massive or massless, the hope is that the dark photon can somehow communicate with the SM as well as connect it to other constituents of the dark side, leading to interesting and potentially observable consequences. These possibilities have received a good deal of theoretical attention in the past few decades [1-22] and stimulated numerous dedicated hunts for dark photons [20-30], albeit still with negative outcomes to date.

Published by the American Physical Society under the terms of the Creative Commons Attribution 4.0 International license. Further distribution of this work must maintain attribution to the author(s) and the published article's title, journal citation, and DOI. Funded by SCOAP ${ }^{3}$.
Most of these efforts have focused on the massive dark photon, $A^{\prime}$, which can couple directly to SM fermions via the renormalizable operator $\epsilon e A_{\mu}^{\prime} J_{\mathrm{EM}}^{\mu}$ involving the electromagnetic current $e J_{\mathrm{EM}}$ and a small constant $\epsilon$ brought about by the kinetic mixing between the dark and SM Abelian gauge fields [18-22]. In the presence of this coupling, $A^{\prime}$ could be produced in the decays or scatterings of ordinary leptons and quarks, including those of hadrons, and it could decay into electrically charged fermions or mesons. Owing to these properties of $A^{\prime}$, the measurements looking for it have been able to acquire limits on $\epsilon$ over various ranges of its mass [19-30].

If the dark photon is massless, the situation is very different but no less interesting. In this case, one can always define a linear combination of the dark and SM U(1) gauge fields that has no renormalizable interactions with the SM and is identified with the massless dark photon [3,6], which we denote by $\bar{\gamma}$. The implications are that it has no direct couplings to SM fermions, in contrast to its massive counterpart, and that therefore restrictions inferred from the aforesaid quests for $A^{\prime}$ do not apply to $\bar{\gamma}$. However, the latter can still affect the SM sector through higherdimensional operators induced by loop diagrams containing particles charged under $\mathrm{U}(1)_{D}$ and also coupled to $\mathrm{SM}$ fields [6-8]. This means that there may be more efficient ways to probe $\bar{\gamma}$, which are worth pursuing and several of which will be explored in this work.

In the absence of other particles beyond the SM lighter than the electroweak scale, the effective interactions of the 
massless dark photon with SM members can be described by operators which respect the SM gauge group and the unbroken $\mathrm{U}(1)_{D}$. At leading order, the couplings of $\bar{\gamma}$ to quarks are of dipole type and given by the gauge-invariant Lagrangian [6]

$$
\mathcal{L}_{\mathrm{NP}}=\frac{1}{\Lambda_{\mathrm{NP}}^{2}}\left(\mathcal{C}_{j k} \overline{q_{j}} \sigma^{\mu \nu} d_{k} H+\mathcal{C}_{j k}^{\prime} \overline{q_{j}} \sigma^{\mu \nu} u_{k} \tilde{H}+\text { H.c. }\right) \bar{F}_{\mu \nu},
$$

where $\Lambda_{\mathrm{NP}}$ denotes an effective heavy mass, the $\mathcal{C}$ s are dimensionless coefficients which are generally complex, $q_{j}$ and $d_{k}\left(u_{k}\right)$ represent a left-handed quark doublet and righthanded down(up)-type quark singlet, respectively, under the $\mathrm{SU}(2)_{L}$ gauge group, $H$ stands for the SM Higgs doublet, $\tilde{H}=i \tau_{2} H^{*}$ with $\tau_{2}$ being the second Pauli matrix, $\bar{F}_{\mu \nu}=\partial_{\mu} \bar{A}_{\nu}-\partial_{\nu} \bar{A}_{\mu}$ is the dark photon's field-strength tensor, $\sigma^{\mu \nu}=i\left[\gamma^{\mu}, \gamma^{\nu}\right] / 2$, and summation over family indices $j, k=1,2,3$ is implicit. Both $\Lambda_{\mathrm{NP}}$ and the $\mathcal{C}$ s depend on the details of the underlying new physics (NP), and in general $\mathcal{C}_{j k}$ and $\mathcal{C}_{j k}^{\prime}$ are not necessarily related to one another.

Here we concern ourselves with flavor-changing neutral current (FCNC) transitions which arise from the operators in Eq. (1), with quarks of the first two generations, and contribute to the decays of hadrons with missing energy. In the SM, the corresponding reactions have an unobserved neutrino pair in the final state and are greatly suppressed, as they proceed from loop diagrams and are subject to the Glashow-Iliopoulos-Maiani mechanism [31,32]. It follows that these FCNC hadron decays may be promising places to seek signs of NP. If $\mathcal{L}_{\mathrm{NP}}$ could impact these processes significantly, a number of them might have rates that are amplified far above their SM expectations to values within the sensitivity ranges of ongoing high-intensity flavor experiments like NA62 [33], KOTO [34], BESIII [35-37], and Belle II [38].

The scenario in which the massless dark photon, $\bar{\gamma}$, possesses flavor-violating interactions with the $d$ and $s$ quarks, via the $\mathcal{C}_{j k}$ portion of $\mathcal{L}_{\mathrm{NP}}$, has already been investigated previously. They translate into the FCNC transitions of strange hadrons with missing energy carried away by $\bar{\gamma}$. As pointed out in Refs. [15,16] ([17]), decays of this kind in the kaon (hyperon) sector have branching fractions that are permitted by current constraints to increase to levels which might be discoverable soon by NA62 and KOTO (BESIII).

In the present paper, we concentrate on the possibility that the massless dark photon has flavor-changing interactions with the $u$ and $c$ quarks, which originate from the $\mathcal{C}_{j k}^{\prime}$ parts in Eq. (1). After electroweak symmetry breaking, in the mass basis of the up-type quarks, we can express the relevant terms as

$$
\mathcal{L}_{u c \bar{\gamma}}=\bar{u}\left(\mathbb{C}+\gamma_{5} \mathbb{C}_{5}\right) \sigma^{\mu \nu} c \bar{F}_{\mu \nu}+\text { H.c. }
$$

and so $\mathbb{C}=\Lambda_{\mathrm{NP}}^{-2}\left(\mathcal{C}_{12}^{\prime}+\mathcal{C}_{21}^{*}\right) v / \sqrt{8}$ and $\mathbb{C}_{5}=\Lambda_{\mathrm{NP}}^{-2}\left(\mathcal{C}_{12}^{\prime}-\right.$ $\left.\mathcal{C}_{21}^{* *}\right) v / \sqrt{8}$ are parameters which have the dimension of inverse mass and are determined by the specifics of the ultraviolet-complete model, with $v \simeq 246 \mathrm{GeV}$ being the Higgs vacuum expectation value. In the next few sections, we analyze the implications for the FCNC decays of several charmed hadrons into two-body final states each consisting of a lighter hadron and $\bar{\gamma}$. The corresponding SM contributions are the three-body modes with a neutrino pair which have tiny branching fractions [32] and hence virtually negligible. On the other hand, the two-body decays with the massless dark photon due to $\mathcal{L}_{u c \bar{\gamma}}$ could have relatively substantial rates, but there have been no direct searches for them yet, as far as we can tell [30]. Therefore, it is hoped that our study will motivate empirical efforts to pursue them, which may shed light on the existence of $\bar{\gamma}$ or at least come up with bounds on $\mathbb{C}$ and $\mathbb{C}_{5}$.

Since a clean environment and sizable luminosity are essential for such endeavors, it is timely that we now have running flavor factories which are potentially well-suited for them, namely, BESIII and Belle II. In these experiments, two charmed hadrons can be created in flavor-conjugate states from each $e^{+} e^{-} \rightarrow c \bar{c}$ event above the pertinent threshold. Moreover, one of the charmed hadrons can be fully reconstructed, and subsequently energy-momentum conservation can be invoked to look for the decay of the other involving missing energy [37-39]. In the future, this procedure would be implemented, likely with much improved sensitivity reach, at the proposed super charm-tau factories $[40,41]$ and Circular Electron Positron Collider (CEPC) operated as a $Z$-boson factory [42].

The rest of the paper is organized in the following way. In Sec. II, we deal with the decays of the lightest charmed hadrons, namely, the pseudoscalar mesons $D^{+}$, $D^{0}$, and $D_{s}^{+}$, into a charmless meson plus $\bar{\gamma}$. For good measure, we include $D^{0} \rightarrow \gamma \bar{\gamma}$, which is also induced by $\mathcal{L}_{u c \bar{\gamma}}$ and emits an ordinary photon, $\gamma$, instead of a meson. In Sec. III, we address the decays of singly charmed baryons $\Lambda_{c}^{+}, \Xi_{c}^{+}$, and $\Xi_{c}^{0}$ into a charmless baryon and $\bar{\gamma}$. Testing these meson and baryon processes would be most feasible at $e^{+} e^{-}$machines, as described in the last paragraph. Charmed hadrons containing more than one heavy quark could undergo analogous reactions, but are either much less likely or too heavy to be produced at existing or upcoming $e^{+} e^{-}$facilities, and so these transitions probably will not be probed for some time. Nevertheless, since the required information is available, for completeness we evaluate in Secs. IV and V the decays of doubly charmed baryon $\Xi_{c c}^{++}$and bottom, charmed pseudoscalar-meson $B_{c}^{+}$, respectively. Finally, in Sec. VI, we give our conclusions. The Appendix supplies extra details on the baryonic matrix elements of the dipole operators in $\mathcal{L}_{u c \bar{\gamma}}$. 


\section{DECAYS OF CHARMED MESONS}

The interactions in $\mathcal{L}_{u c \bar{\gamma}}$ give rise to the FCNC decays of the lightest charmed mesons into a charmless meson and the dark photon. Given that the decay of a spinless particle into another spinless particle plus a massless gauge boson is forbidden by angular-momentum conservation and gauge invariance, in most of this section we consider modes where the initial particle is the charmed pseudoscalar meson $D^{+}$or $D^{0}$ or the charmed, strange pseudoscalar meson $D_{s}^{+}$, whereas the daughter particles consist of a charmless vector meson and $\bar{\gamma}$. In particular, we examine
$D^{+} \rightarrow \rho^{+} \bar{\gamma}, D^{0} \rightarrow \rho^{0} \bar{\gamma}, D^{0} \rightarrow \omega \bar{\gamma}$, and $D_{s}^{+} \rightarrow K^{*+} \bar{\gamma}$. As for $D^{0} \rightarrow \gamma \bar{\gamma}$, we will treat it toward the end of the section.

For $D^{+} \rightarrow \rho^{+} \bar{\gamma}$, the amplitude $\mathcal{M}_{D^{+} \rightarrow \rho^{+} \bar{\gamma}}$ contains the mesonic matrix elements $\left\langle\rho^{+}\left|\bar{u} \sigma^{\mu \nu} c\right| D^{+}\right\rangle$and $\left\langle\rho^{+}\left|\bar{u} \sigma^{\mu \nu} \gamma_{5} c\right| D^{+}\right\rangle$, the general formulas for which are long known in the literature [43-45]. In $\mathcal{M}_{D^{+} \rightarrow \rho^{+} \bar{\gamma}}$, these matrix elements are to be contracted with the outgoing dark photon's momentum $\bar{q}$ and polarization vector $\bar{\varepsilon}$. These 2 four-vectors fulfill the gauge requirement $\bar{\varepsilon} \cdot \bar{q}=0$ and the masslessness condition $\bar{q}^{2}=0$ owing to $\bar{\gamma}$ being on shell. Accordingly, we can write

$$
\begin{aligned}
\left\langle\rho^{+}(k)\left|\bar{u} \sigma^{\mu \nu} c\right| D^{+}(k+\bar{q})\right\rangle \bar{\varepsilon}_{\mu}^{*} \bar{q}_{\nu} & =2 i f_{D^{+} \rho^{+}} \epsilon^{\eta \tau \mu \nu} \varepsilon_{\eta}^{*} k_{\tau} \bar{\varepsilon}_{\mu}^{*} \bar{q}_{\nu}, \\
\left\langle\rho^{+}(k)\left|\bar{u} \sigma^{\mu \nu} \gamma_{5} c\right| D^{+}(k+\bar{q})\right\rangle \bar{\varepsilon}_{\mu}^{*} \bar{q}_{\nu} & =2 f_{D^{+} \rho^{+}}\left(\varepsilon^{*} \cdot \bar{q} \bar{\varepsilon}^{*} \cdot k-\varepsilon^{*} \cdot \bar{\varepsilon}^{*} k \cdot \bar{q}\right),
\end{aligned}
$$

where $k$ and $\varepsilon$ stand for the $\rho^{+}$momentum and polarization vector, respectively, $k+\bar{q}$ is the $D^{+}$momentum, and the same constant $f_{D^{+} \rho^{+}}$parametrizes form factor effects at $\bar{q}^{2}=0$ in the two equations, which are related [43] by virtue of the identity $2 i \sigma^{\eta \kappa} \gamma_{5}=\epsilon^{\eta \kappa \nu \tau} \sigma_{\nu \tau}$. They lead to

$$
\mathcal{M}_{D^{+} \rightarrow \rho^{+} \bar{\gamma}}=4 f_{D^{+} \rho^{+}}\left[\epsilon^{\eta \kappa \nu \tau} \varepsilon_{\eta}^{*} \bar{\varepsilon}_{\kappa}^{*} k_{\nu} \bar{q}_{\tau} \mathbb{C}+i\left(\varepsilon^{*} \cdot \bar{q} \bar{\varepsilon}^{*} \cdot k-\varepsilon^{*} \cdot \bar{\varepsilon}^{*} k \cdot \bar{q}\right) \mathbb{C}_{5}\right],
$$

which is $\mathrm{U}(1)_{D}$-gauge invariant and from which we obtain the branching fraction

$$
\mathcal{B}\left(D^{+} \rightarrow \rho^{+} \bar{\gamma}\right)=\frac{\tau_{D^{+}} f_{D^{+} \rho^{+}}^{2}\left(m_{D^{+}}^{2}-m_{\rho^{+}}^{2}\right)^{3}}{2 \pi m_{D^{+}}^{3}}\left(|\mathbb{C}|^{2}+\left|\mathbb{C}_{5}\right|^{2}\right),
$$

where $\tau_{D^{+}}$represents the lifetime of $D^{+}$and $m_{\mathcal{X}}$ denotes the mass of $\mathcal{X}$. The corresponding quantities for $D^{0} \rightarrow \rho^{0} \bar{\gamma}, \omega \bar{\gamma}$ and $D_{s}^{+} \rightarrow K^{*+} \bar{\gamma}$ have analogous expressions.

For numerical calculations, we employ

$$
f_{D^{+} \rho^{+}}=\sqrt{2} f_{D^{0} \rho^{0}}=0.658_{-0.031}^{+0.038}, \quad f_{D^{0} \omega}=0.610_{-0.030}^{+0.036}, \quad f_{D_{s}^{+} K^{*+}}=0.639_{-0.044}^{+0.042},
$$

which have been estimated in Ref. [45] with light-cone sum rules in the framework of heavy-quark effective field theory. The relation between $f_{D^{+} \rho^{+}}$and $f_{D^{0} \rho^{0}}$ in Eq. (6) follows from the quark flavor contents $\rho^{+} \sim u \bar{d}$ and $\rho^{0} \sim(u \bar{u}-d \bar{d}) / \sqrt{2}$. For comparison, an earlier analysis within a constituent quark model [44] yielded $f_{D^{+} \rho^{+}}=$ 0.66 and $f_{D_{s}^{+} K^{*+}}=0.71$ with uncertainties of around $10 \%$, which are compatible with their newer counterparts in Eq. (6). Additional input parameters are the empirical $D^{+, 0}$ and $D_{s}^{+}$lifetimes and masses and the light meson masses, namely, $\quad m_{\rho^{+}}=775.11(34), \quad m_{\rho^{0}}=775.26(25), \quad m_{\omega}=$ $782.65(12)$, and $m_{K^{*+}}=895.5(8)$, in units of $\mathrm{MeV}$, all from Ref. [30]. Thus, the only remaining unknowns are the coefficients $\mathbb{C}$ and $\mathbb{C}_{5}$, which depend on the details of the NP model.

These processes can serve as valuable probes of the massless dark photon if their rates are not highly suppressed. Clearly, this can happen provided that one or both of $\mathbb{C}$ and $\mathbb{C}_{5}$ are not too small in size. At the moment, there are no model-independent restrictions on them, as there are still no data on these charmed-hadron decays [30]. It turns out that there is at least one NP model in the recent literature [7] which offers some non-negligible viable values of these coefficients. Those numbers can then serve as benchmarks to illustrate how these charmed-hadron decays may shed light on the dark photon's existence.

In the NP scenario of Ref. [7], the $c \rightarrow u \bar{\gamma}$ operators arise from loop diagrams involving massive fermions which are singlets under the SM gauge group and heavy new scalar bosons which carry some of the SM gauge charges. The new fermions and bosons are all charged under $\mathrm{U}(1)_{D}$ and have Yukawa-like interactions with the $u$ and $c$ quarks. This allows for the construction of the dimension-five operators in Eq. (2). Since in this paper we are mainly interested in the implications of these effective interactions for the charmed-hadron decays, we will not dwell further on the specifics of the underlying NP model. Rather, we will simply adopt the relevant results available from Ref. [7] at 
face value and apply them to the determination of the rates of the charmed-hadron processes.

Particularly, as elaborated in Ref. [7], the branching fraction of the inclusive transition $c \rightarrow u \bar{\gamma}$ could be as much as about $10^{-4}$, with the pertinent constraints, from dark matter and vacuum stability considerations, having been taken into account. Accordingly, for the purposes of our study, we can take $\mathcal{B}(c \rightarrow u \bar{\gamma})<5 \times 10^{-5}$, which translates into ${ }^{1}$

$$
|\mathbb{C}|^{2}+\left|\mathbb{C}_{5}\right|^{2}<\frac{1.9 \times 10^{-16}}{\mathrm{GeV}^{2}}
$$

It might seem that this is very optimistic because $\mathcal{B}(c \rightarrow u \bar{\gamma})$ in Ref. [7] ranges from $10^{-13}$ to $10^{-4}$, as Table VI and Fig. 6 therein show. However, given that direct-search bounds on the couplings are currently absent, it is possible that there are other NP models which can generate numbers larger than that in Eq. (7). Furthermore, model independently, $|\mathbb{C}|^{2}+\left|\mathbb{C}_{5}\right|^{2} \sim 10^{-15} \mathrm{GeV}^{2}$ would even still be permitted at present, being compatible with the caps on the branching fractions of yet-unobserved $D^{+, 0}$ and $D_{s}^{+}$decays. ${ }^{2}$ Therefore, what we have in Eq. (7) is a reasonable reference value with which to make benchmark predictions that are potentially testable by searches in the near future.

The rates of all the decays which will be discussed shortly are proportional to $|\mathbb{C}|^{2}+\left|\mathbb{C}_{5}\right|^{2}$. Consequently, if hunts for these transitions come up with limits stronger than the branching fractions we compute below based on Eq. (7), the implied restraints are germane solely to this combination of the coefficients. To investigate $\mathbb{C}$ and $\mathbb{C}_{5}$ separately would require measuring more complicated observables, such as the angular distributions of the decay products, but this step will be worth taking only after one or more of these decays are discovered.

Incorporating the central values of the aforementioned input parameters and Eq. (7) into the meson branching fractions, we arrive at

$$
\begin{aligned}
\mathcal{B}\left(D^{+} \rightarrow \rho^{+} \bar{\gamma}\right) & =4.04 \times 10^{11} \tilde{\mathrm{C}}^{2}<7.7 \times 10^{-5}, \\
\mathcal{B}\left(D^{0} \rightarrow \rho^{0} \bar{\gamma}\right) & =7.88 \times 10^{10} \tilde{\mathrm{C}}^{2}<1.5 \times 10^{-5}, \\
\mathcal{B}\left(D^{0} \rightarrow \omega \bar{\gamma}\right) & =1.34 \times 10^{11} \tilde{\mathrm{C}}^{2}<2.5 \times 10^{-5}, \\
\mathcal{B}\left(D_{s}^{+} \rightarrow K^{*+} \bar{\gamma}\right) & =1.89 \times 10^{11} \tilde{\mathrm{C}}^{2}<3.6 \times 10^{-5},
\end{aligned}
$$

\footnotetext{
${ }^{1}$ This has been found by using $\mathcal{B}(c \rightarrow u \bar{\gamma})=12 \pi^{2} \mathcal{B}(c \rightarrow$ $\left.\ell^{+} X\right)_{\exp }\left(1 /\left|\Lambda_{L}\right|^{2}+1 /\left|\Lambda_{R}\right|^{2}\right) /\left[G_{\mathrm{F}}^{2} m_{c}^{2}\left|V_{\mathrm{cs}}\right|^{2} \mathrm{f}_{1}\left(m_{s} / m_{c}\right)\right] \quad$ upon adding the factor $\pi^{2}$ missing from Eq. (88) in [7], with $\mathcal{B}(c \rightarrow$ $\left.\ell^{+} X\right)_{\exp }=0.096$ from the $D^{+}$listing in [30], $G_{\mathrm{F}}$ being the Fermi constant, $m_{c}=1.67 \mathrm{GeV},\left|V_{c s}\right|=0.986$ [7], $\mathrm{f}_{1}(x)=1-8 x^{2}+$ $8 x^{6}-x^{8}-24 x^{4} \ln x$, and $m_{s} / m_{c} \simeq 0.0853$ [30], along with the relation $1 /\left|\Lambda_{L}\right|^{2}+1 /\left|\Lambda_{R}\right|^{2}=8|\mathbb{C}|^{2}+8\left|\mathbb{C}_{5}\right|^{2}$.

${ }^{2}$ With $|\mathbb{C}|^{2}+\left|\mathbb{C}_{5}\right|^{2}=10^{-15} \mathrm{GeV}^{2}$, the branching-fraction maxima in Eq. (8) would be $\sim 5$ times greater. The increased results are well under the caps on the branching fractions of yetunobserved decay channels of $D^{+, 0}$ and $D_{s}^{+}$, which exceed $1 \%$, as can be inferred from the data on their observed ones [30].
}

where, for conciseness, we have defined

$$
\tilde{\mathrm{C}}^{2}=\left(|\mathbb{C}|^{2}+\left|\mathbb{C}_{5}\right|^{2}\right) \mathrm{GeV}^{2} .
$$

Interestingly, these numbers are comparable to the latest limit $\mathcal{B}\left(D^{0} \rightarrow\right.$ invisibles $)<9.4 \times 10^{-5}$ at $90 \%$ confidence level [30] set by the Belle Collaboration [39]. This suggests that one or more of the results in Eq. (8) may be within reach of the ongoing BESIII [36] and Belle II [38] experiments. ${ }^{3}$ If not, significantly improved probes would probably be available from proposed facilities like the super charm-tau factories [40,41] and CEPC [42].

For $D^{0} \rightarrow \gamma \bar{\gamma}$, we need the matrix elements

$$
\begin{aligned}
\left\langle\gamma(k)\left|\bar{u} \sigma^{\mu \nu} c\right| D^{0}(k+\bar{q})\right\rangle \bar{\varepsilon}_{\mu}^{*} \bar{q}_{\nu}= & i e f_{D^{0} \gamma} \epsilon^{\eta \tau \mu \nu} \breve{\varepsilon}_{\eta}^{*} k_{\tau} \bar{\varepsilon}_{\mu}^{*} \bar{q}_{\nu}, \\
\left\langle\gamma(k)\left|\bar{u} \sigma^{\mu \nu} \gamma_{5} c\right| D^{0}(k+\bar{q})\right\rangle \bar{\varepsilon}_{\mu}^{*} \bar{q}_{\nu}= & e f_{D^{0} \gamma}\left(\breve{\varepsilon}^{*} \cdot \bar{q} \bar{\varepsilon}^{*} \cdot k\right. \\
& \left.-\check{\varepsilon}^{*} \cdot \bar{\varepsilon}^{*} k \cdot \bar{q}\right),
\end{aligned}
$$

which have also been considered in the literature [46,47] and involve the electric charge $e$, the photon's polarization vector $\check{\varepsilon}$, and the form-factor parameter $f_{D^{0} \gamma}$. Therefore, the decay amplitude satisfies both the $\mathrm{U}(1)_{D}$ and electromagnetic gauge symmetries. The corresponding branching fraction is

$$
\mathcal{B}\left(D^{0} \rightarrow \gamma \bar{\gamma}\right)=\frac{\alpha_{\mathrm{e}}}{2} \tau_{D^{0}} f_{D^{0} \gamma}^{2} m_{D^{0}}^{3}\left(|\mathbb{C}|^{2}+\left|\mathbb{C}_{5}\right|^{2}\right),
$$

where $\alpha_{\mathrm{e}}=e^{2} /(4 \pi)=1 / 137$. Hence, including $f_{D^{0} \gamma}=$ 0.33 from Ref. [47] and Eq. (7) leads to

$$
\mathcal{B}\left(D^{0} \rightarrow \gamma \bar{\gamma}\right)=1.61 \times 10^{9} \tilde{\mathrm{C}}^{2}<3.1 \times 10^{-7} .
$$

This is roughly 2 orders of magnitude below its counterparts in Eq. (8), which is due to the presence of $\alpha_{\mathrm{e}}$.

\section{DECAYS OF SINGLY CHARMED BARYONS}

In the baryon sector, the $c \rightarrow u \bar{\gamma}$ transition in Eq. (2) also causes charmed baryons to undergo $\Delta C=1$ decays into a lighter baryon plus the dark photon. In this section, we focus on the reactions of the singly charmed baryons $\Lambda_{c}^{+}$, $\Xi_{c}^{+}$, and $\Xi_{c}^{0}$, which have spin parity $J^{P}=1 / 2^{+}$, make up a flavor SU(3) antitriplet, and decay weakly [30]. We examine in particular the modes $\Lambda_{c}^{+} \rightarrow p \bar{\gamma}$ and $\Xi_{c}^{+, 0} \rightarrow \Sigma^{+, 0} \bar{\gamma}$, which BESIII and Belle II could also pursue.

\footnotetext{
${ }^{3}$ We may take the Belle finding on $\mathcal{B}\left(D^{0} \rightarrow\right.$ invisibles $)$ as a guide for making a rough guess on whether Eq. (8) may be accessible to Belle II. The detector performance of Belle II is similar to that of Belle, but the accumulated integrated luminosity will be 50 times bigger [38]. Thus, if the reconstruction efficiency of the $\rho$ meson is $20 \%$ or higher at Belle II, we expect it to have the sensitivity $\mathcal{B}(D \rightarrow \rho \bar{\gamma}) \sim 9.4 \times 10^{-5} /(0.2 \times 50) \sim 10^{-5}$ or better, indicating that it may be able to test the results in Eq. (8).
} 
To derive the amplitude $\mathcal{M}_{\Lambda_{c}^{+} \rightarrow p \bar{\gamma}}$ for $\Lambda_{c}^{+} \rightarrow p \bar{\gamma}$ from the short-distance interaction described by $\mathcal{L}_{u c \bar{\gamma}}$, we need to know the matrix elements $\left\langle p\left|\bar{u} \sigma^{\mu \nu} c\right| \Lambda_{c}^{+}\right\rangle$and $\left\langle p\left|\bar{u} \sigma^{\mu \nu} \gamma_{5} c\right| \Lambda_{c}^{+}\right\rangle$. Contracting them with the dark photon's momentum $\bar{q}$ and polarization vector $\bar{\varepsilon}$, we can write

$$
\left\langle p\left|\bar{u} \sigma^{\mu \nu}\left(1, \gamma_{5}\right) c\right| \Lambda_{c}^{+}\right\rangle \bar{\varepsilon}_{\mu}^{*} \bar{q}_{\nu}=f_{\Lambda_{c}^{+} p} \overline{\mathrm{U}_{p}} \sigma^{\mu \nu}\left(1, \gamma_{5}\right) \mathrm{U}_{\Lambda_{c}} \bar{\varepsilon}_{\mu}^{*} \bar{q}_{\nu},
$$

where $\mathrm{U}_{\Lambda_{c}}$ and $\mathrm{U}_{p}$ stand for the Dirac spinors of the baryons and the same constant $f_{\Lambda_{c}^{+} p}$, which encodes form-factor effects at $\bar{q}^{2}=0$, enters both of the matrix elements, as explained in the Appendix. It follows that

$$
\mathcal{M}_{\Lambda_{c}^{+} \rightarrow p \bar{\gamma}}=2 f_{\Lambda_{c}^{+} p} \overline{\mathrm{U}_{p}}\left(\mathbb{C}+\gamma_{5} \mathbb{C}_{5}\right) i \sigma^{\mu \nu} \mathrm{U}_{\Lambda_{c}} \bar{\varepsilon}_{\mu}^{*} \bar{q}_{\nu},
$$

which fulfills $\mathrm{U}(1)_{D}$-gauge invariance, and so the branching fraction is

$$
\mathcal{B}\left(\Lambda_{c}^{+} \rightarrow p \bar{\gamma}\right)=\frac{\tau_{\Lambda_{c}^{+}} f_{\Lambda_{c}^{+} p}^{2}\left(m_{\Lambda_{c}^{+}}^{2}-m_{p}^{2}\right)^{3}}{2 \pi m_{\Lambda_{c}^{+}}^{3}}\left(|\mathbb{C}|^{2}+\left|\mathbb{C}_{5}\right|^{2}\right),
$$

where $\tau_{\Lambda_{c}^{+}}$is the $\Lambda_{c}^{+}$lifetime. Numerically, we adopt $f_{\Lambda_{c}^{+} p}=0.50(1 \pm 0.07)$ from the lattice QCD calculation in Ref. [48]. Incorporating its central value and those of $\tau_{\Lambda_{c}^{+}}, m_{\Lambda_{c}^{+}}$, and $m_{p}$ from Ref. [30] into Eq. (15), we then translate the limit in Eq. (7) into

$$
\mathcal{B}\left(\Lambda_{c}^{+} \rightarrow p \bar{\gamma}\right)=8.31 \times 10^{10} \tilde{\mathrm{C}}^{2}<1.6 \times 10^{-5} .
$$

If instead $f_{\Lambda_{c}^{+} p}=0.38(1 \pm 0.1)$, evaluated in the relativistic quark model [49], we would get a lower result, $\mathcal{B}\left(\Lambda_{c}^{+} \rightarrow p \bar{\gamma}\right)<9.1 \times 10^{-6}$. These different numbers indicate the degree of uncertainty in the prediction.

In the case of $\Xi_{c}^{0} \rightarrow \Sigma^{0} \bar{\gamma}$, we employ the form-factor parameter $f_{\Xi_{c}^{0} \Sigma^{0}}=0.46 \pm 0.12$, which has been estimated in the framework of light-cone QCD sum rules [50]. Assuming isospin symmetry, we also have $f_{\Xi_{c}^{+} \Sigma^{+}}=$ $f_{\Xi_{c}^{0} \Sigma^{0}}$ for $\Xi_{c}^{+} \rightarrow \Sigma^{+} \bar{\gamma}$. Their branching fractions have expressions obtainable from Eq. (15), with suitable modifications. With the central values of $f_{\Xi_{c}^{0} \Sigma^{0}}$ and the measured $\Xi_{c}^{+, 0}$ lifetimes and relevant baryon masses [30], along with Eq. (7), we then arrive at

$$
\begin{aligned}
\mathcal{B}\left(\Xi_{c}^{+} \rightarrow \Sigma^{+} \bar{\gamma}\right) & =1.54 \times 10^{11} \tilde{\mathrm{C}}^{2}<2.9 \times 10^{-5}, \\
\mathcal{B}\left(\Xi_{c}^{0} \rightarrow \Sigma^{0} \bar{\gamma}\right) & =3.90 \times 10^{10} \tilde{\mathrm{C}}^{2}<7.4 \times 10^{-6} .
\end{aligned}
$$

The difference between them is attributable mainly to $\tau_{\Xi_{c}^{+}}=3.9 \tau_{\Xi_{c}^{0}}$.

\section{DECAYS OF DOUBLY CHARMED BARYON}

In this and the next sections, we look at the impact of $\mathcal{L}_{u c \bar{\gamma}}$ on the decays of hadrons containing two heavy quarks, specifically $\Xi_{c c}^{++}$and $B_{c}^{+}$, respectively. As $\mathrm{LHCb}$ will be the primary facility that produces them for the foreseeable future [51], and they are either much less likely or too heavy to be produced at ongoing $e^{+} e^{-}$facilities, these transitions probably will not be probed any time soon. We include them here for completeness, as the ingredients needed to treat them are already on hand.

Only one doubly charmed baryon, $\Xi_{c c}^{++}$, has been discovered so far [52], with its lifetime and mass now also determined [30]. We take its spin parity, which is not yet established experimentally, to be $J^{P}=1 / 2^{+}$based on quark-model expectations [52]. Here we consider the decay channels $\Xi_{c c}^{++} \rightarrow \Sigma_{c}(2455)^{++} \bar{\gamma}$ and $\Xi_{c c}^{++} \rightarrow \Sigma_{c}(2520)^{++} \bar{\gamma}$ arising from $\mathcal{L}_{c u \bar{\gamma}}$, as some information on the pertinent baryonic form factors has recently become available [53].

Since $\Sigma_{c}(2455)^{++}$also has $J^{P}=1 / 2^{+}$[30], the branching fraction of $\Xi_{c c}^{++} \rightarrow \Sigma_{c}(2455)^{++} \bar{\gamma}$ is analogous to that in Eq. (15), namely,

$$
\begin{aligned}
& \mathcal{B}\left(\Xi_{c c}^{++} \rightarrow \Sigma_{c}(2455)^{++} \bar{\gamma}\right) \\
& \quad=\frac{\tau_{\Xi_{c c}^{++}} f_{\Xi_{c c} \Sigma_{c}(2455)}^{2}\left(m_{\Xi_{c c}}^{2}-m_{\Sigma_{c}(2455)^{++}}^{2}\right)^{3}}{2 \pi m_{\Xi_{c c}}^{3}}\left(|\mathbb{C}|^{2}+\left|\mathbb{C}_{5}\right|^{2}\right),
\end{aligned}
$$

where $m_{\Xi_{c c}} \equiv m_{\Xi_{c c}^{+}}$. In this equation, we use the formfactor parameter $f_{\Xi_{c c} \Sigma_{c}(2455)}=-0.798$ which has been computed in the light-front quark model with an uncertainty of several percent [53]. With the central values of the empirical $\Xi_{c c}^{++}$lifetime and mass and $m_{\Sigma_{c}(2455)^{++}}=$ 2453.97 MeV from Ref. [30], plus the bound in Eq. (7), we then find

$\mathcal{B}\left(\Xi_{c c}^{++} \rightarrow \Sigma_{c}(2455)^{++} \bar{\gamma}\right)=2.96 \times 10^{11} \tilde{\mathrm{C}}^{2}<5.6 \times 10^{-5}$.

In the second channel, the daughter baryon $\Sigma_{c}(2520)^{++}$ has spin parity $J^{P}=3 / 2^{+}$[30]. As discussed in the Appendix, the baryonic matrix elements for this reaction are

$$
\begin{aligned}
& \left\langle\Sigma_{c}(2520)^{++}\left|\bar{u} i \sigma^{\mu \nu}\left(1, \gamma_{5}\right) c\right| \Xi_{c c}^{++}\right\rangle \bar{\varepsilon}_{\mu}^{*} \bar{q}_{\nu} \\
& =\overline{\mathbb{U}}_{\Sigma_{c}}^{\kappa}\left(\gamma_{5}, 1\right)\left[\left(\gamma^{\tau} \bar{\varepsilon}_{\tau}^{*} \bar{q}_{\kappa}-\bar{\varepsilon}_{\kappa}^{*} \bar{q}\right) \mathcal{F}+\frac{\bar{q}_{\kappa} \gamma^{\tau} \bar{\varepsilon}_{\tau}^{*} \bar{q} \tilde{\mathcal{F}}}{m_{\Xi_{c c}}}\right] \mathrm{U}_{\Xi_{c c}},
\end{aligned}
$$

where $\mathbb{U}_{\Sigma_{c}}^{\kappa}$ denotes the Rarita-Schwinger spinor of $\Sigma_{c}(2520)^{++}$and the constants $\mathcal{F}$ and $\tilde{\mathcal{F}}$ parametrize form-factor effects at $\bar{q}^{2}=0$. We can then write the decay amplitude 


$$
\begin{aligned}
& \mathcal{M}_{\Xi_{c c}^{++} \rightarrow \Sigma_{c}(2520)^{++} \bar{\gamma}} \\
& =2 \overline{\mathbb{U}}_{\Sigma_{c}}^{\kappa}\left(\gamma_{5} \mathbb{C}+\mathbb{C}_{5}\right)\left[\left(\gamma^{\tau} \bar{\varepsilon}_{\tau}^{*} \bar{q}_{\kappa}-\bar{\varepsilon}_{\kappa}^{*} \not{\mathcal{q}}\right) \mathcal{F}+\frac{\bar{q}_{\kappa} \gamma^{\tau} \bar{\varepsilon}_{\tau}^{*} \not{\mathcal{q}} \tilde{\mathcal{F}}}{m_{\Xi_{c c}}}\right] \mathrm{U}_{\Xi_{c c}},
\end{aligned}
$$

which is $\mathrm{U}(1)_{D}$-gauge invariant and leads to the branching fraction, ${ }^{4}$

$$
\begin{aligned}
\mathcal{B}\left(\Xi_{c c}^{++}\right. & \left.\rightarrow \Sigma_{c}(2520)^{++} \bar{\gamma}\right) \\
= & \frac{\tau_{\Xi_{c c}^{++}}^{++} \Delta^{8} \mathcal{F}^{2}}{12 \pi m_{\Xi_{c c}}^{3} m_{\Sigma_{c}}^{2}}\left(1+\frac{4 m_{\Sigma_{c}}^{2}}{\Delta^{2}}+\frac{2 \tilde{\mathcal{F}}}{\mathcal{F}}+\frac{\Delta^{2} \tilde{\mathcal{F}}^{2}}{m_{\Xi_{c c}}^{2} \mathcal{F}^{2}}\right) \\
& \times\left(|\mathbb{C}|^{2}+\left|\mathbb{C}_{5}\right|^{2}\right),
\end{aligned}
$$

where $m_{\Sigma_{c}} \equiv m_{\Sigma_{c}(2520)^{++}}$and $\Delta=\left(m_{\Xi_{c c}}^{2}-m_{\Sigma_{c}}^{2}\right)^{1 / 2}$. Adopting $\mathcal{F}=-0.635$ and $\tilde{\mathcal{F}}=0.330$, which have been estimated in the light-front quark model with uncertainties of several percent [53], with $m_{\Sigma_{c}}=2518.41 \mathrm{MeV}$ [30] and Eq. (7), we then obtain

$$
\mathcal{B}\left(\Xi_{c c}^{++} \rightarrow \Sigma_{c}(2520)^{++} \bar{\gamma}\right)=1.12 \times 10^{11} \tilde{\mathrm{C}}^{2}<2.1 \times 10^{-5} .
$$

\section{DECAY OF BOTTOM, CHARMED MESON}

The $c \rightarrow u \bar{\gamma}$ transition can also bring about the decay of the bottom, charmed pseudoscalar meson $B_{c}^{+}$into the bottom charged vector meson $B^{*+}$ and the dark photon. Consequently, the amplitude for $B_{c}^{+} \rightarrow B^{*+} \bar{\gamma}$ and its branching fraction are similar to the corresponding quantities associated with $D^{+} \rightarrow \rho^{+} \bar{\gamma}$, given in Eqs. (4) and (5). Especially, based on the latter, we have

$\mathcal{B}\left(B_{c}^{+} \rightarrow B^{*+} \bar{\gamma}\right)=\frac{\tau_{B_{c}^{+}} f_{B_{c} B_{u}^{*}}^{2}\left(m_{B_{c}^{+}}^{2}-m_{B^{*+}}^{2}\right)^{3}}{2 \pi m_{B_{c}^{+}}^{3}}\left(|\mathbb{C}|^{2}+\left|\mathbb{C}_{5}\right|^{2}\right)$.

To evaluate this, we employ the known $B_{c}^{+}$lifetime and mass and $m_{B^{*+}}=5324.70(22) \mathrm{MeV}$ from Ref. [30], as well as $f_{B_{c} B_{u}^{*}}=0.23 \pm 0.04$ which has been calculated in Ref. [55] with QCD sum rules and is in agreement with the older result $f_{B_{c} B_{u}^{*}}=0.24$ of Ref. [56] from an application of the constituent quark model of Ref. [57]. Combining their central values and Eq. (7) with Eq. (24) then yields

$$
\mathcal{B}\left(B_{c}^{+} \rightarrow B^{*+} \bar{\gamma}\right)=3.54 \times 10^{10} \tilde{\mathrm{C}}^{2}<6.7 \times 10^{-6} .
$$

\footnotetext{
${ }^{4}$ It is worth noting that Eqs. (21) and (22) can be shown to be consistent in form with the amplitude and branching fraction, respectively, of $\Lambda_{b} \rightarrow \Lambda(1520) \gamma$, which involves the ordinary photon, in [54], after interchanging the parity-conserving and -violating terms in the amplitude, as $\Sigma_{c}(2520)^{++}$and $\Lambda(1520)$ are opposite in parity.
}

\section{CONCLUSIONS}

If a new $\mathrm{U}(1)$ gauge symmetry under which all SM particles are singlets exists and is spontaneously broken, the accompanying dark photon has a nonzero mass and may undergo direct renormalizable interactions with SM fermions due to kinetic mixing between the dark and SM U(1) gauge fields. This kind of dark photon has been the subject of many searches in recent years, which have come up empty so far. If the extra U(1) gauge group stays unbroken, the associated dark photon is massless instead and can couple to SM members only through higher-dimensional operators, implying that it would have evaded the aforesaid hunts for its massive counterpart. Therefore, it is crucial that upcoming endeavors to seek dark photons take into account the possibility that they are massless, in which case they may have consequential FCNC interactions with SM fermions.

In this paper, we explore the latter scenario, particularly that where the massless dark photon has nonnegligible flavor-changing dipole-type couplings to the $u$ and $c$ quarks. If occurring in nature, these interactions will induce the FCNC decays of charmed hadrons into a lighter hadron plus missing energy carried away by the dark photon. We propose to pursue a number of such $\Delta C=1$ reactions, focusing on those in which the parent particles are the charmed pseudoscalar mesons $D^{+, 0}$ and $D_{s}^{+}$, the singly charmed baryons $\Lambda_{c}^{+}, \Xi_{c}^{+}$, and $\Xi_{c}^{0}$, the doubly-charmed baryon $\Xi_{c c}^{++}$, and the bottom, charmed pseudoscalar meson $B_{c}^{+}$. We also consider $D^{0} \rightarrow \gamma \bar{\gamma}$, which has the standard photon besides $\bar{\gamma}$ in the final state. In the context of a simplified new-physics model, we show that some of the $D^{+, 0}, D_{s}^{+}$, and singly charmedbaryon modes have branching fractions that are permitted by present constraints to be as large as several times $10^{-5}$, which may be within the sensitivity reach of BESIII and Belle II. Since the same one or two $c \rightarrow$ $u \bar{\gamma}$ operators are responsible for all these decays, detecting one of them automatically implies specific predictions for the others, allowing for additional empirical tests on the dark photon. Conversely, measuring a bound on one of the decays will translate into expected bounds on the others. The results of this work will hopefully help motivate dedicated attempts to look for massless dark photons via charmed-hadron processes. These efforts will be complementary to the quests for the massive dark photon, which is phenomenologically quite distinct from the massless one.

\section{ACKNOWLEDGMENTS}

We would like to thank Cong Geng, Hai-Bo Li, and Min-Zu Wang for information on experimental matters. This research was supported in part by the MOST (Grant No. MOST 106-2112-M-002-003-MY3). 


\section{APPENDIX: BARYONIC MATRIX ELEMENTS OF $\bar{u} \sigma^{\mu \nu}\left(1, \gamma_{5}\right) c$}

For the $\Delta C=1$ transition between baryons $\mathfrak{B}$ and $\mathfrak{B}^{\prime}$ with spin parity $J^{P}=1 / 2^{+}$, the baryonic matrix element of $\bar{u} \sigma^{\mu \nu} c$ has the general expression [58,59] which is as follows:

$$
\left\langle\mathfrak{B}^{\prime}(k)\left|\bar{u} \sigma^{\mu \nu} c\right| \mathfrak{B}(k+\bar{q})\right\rangle=\overline{\mathrm{U}^{\prime}}\left(\sigma^{\mu \nu} f_{1}-\gamma^{[\mu} k^{\nu]} i f_{2}-\gamma^{[\mu} \bar{q}^{\nu]} i f_{3}-k^{[\mu} \bar{q}^{\nu]} i f_{4}\right) \mathrm{U}
$$

where $k+\bar{q}$ and $k$ are the momenta of $\mathfrak{B}$ and $\mathfrak{B}^{\prime}$, respectively, $\mathrm{U}$ and $\mathrm{U}^{\prime}$ designate their Dirac spinors, $f_{1,2,3,4}$ denote form factors which are functions of $\bar{q}^{2}$, and $\mathrm{X}^{[\mu} \mathrm{Y}^{\nu]} \equiv \mathrm{X}^{\mu} \mathrm{Y}^{\nu}-\mathrm{X}^{\nu} \mathrm{Y}^{\mu}$. With the aid of the identity $2 i \sigma_{\eta \kappa} \gamma_{5}=\epsilon_{\eta \kappa \nu \tau} \sigma^{\nu \tau}$ for $\epsilon_{0123}=+1$, we can derive from Eq. (A1) the corresponding matrix element of $\bar{u} \sigma_{\eta \kappa} \gamma_{5} c$,

$$
\left\langle\mathfrak{B}^{\prime}(k)\left|\bar{u} \sigma_{\eta \kappa} \gamma_{5} c\right| \mathfrak{B}(k+\bar{q})\right\rangle=\overline{\mathrm{U}^{\prime}}\left[\sigma_{\eta \kappa} \gamma_{5} f_{1}-\epsilon_{\eta \kappa \nu \tau}\left(\gamma^{[\nu} k^{\tau]} \frac{f_{2}}{2}+\gamma^{\nu} \bar{q}^{\tau} f_{3}+k^{\nu} \bar{q}^{\tau} f_{4}\right)\right] \mathrm{U} .
$$

In the treatment of the amplitude for $\mathfrak{B} \rightarrow \mathfrak{B}^{\prime} \bar{\gamma}$, the matrix elements in Eqs. (A1) and (A2) are to be contracted with the

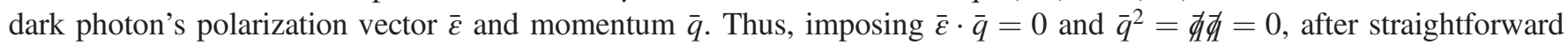
algebra, we arrive at

$$
\begin{aligned}
\left\langle\mathfrak{B}^{\prime}(k)\left|\bar{u} \sigma^{\mu \nu} c\right| \mathfrak{B}(k+\bar{q})\right\rangle \bar{\varepsilon}_{\mu}^{*} \bar{q}_{\nu} & =\overline{\mathrm{U}^{\prime}}\left\{\sigma^{\mu \nu} f_{1}^{(0)}-i\left[\gamma^{\mu} \gamma^{\nu}(\not k+\not k)-\not k \gamma^{\mu} \gamma^{\nu}\right] \frac{f_{2}^{(0)}}{2}\right\} \mathrm{U} \bar{\varepsilon}_{\mu}^{*} \bar{q}_{\nu} \\
& =f_{\mathfrak{B}^{\prime}} \overline{\mathrm{U}}^{\prime} \sigma^{\mu \nu} \mathrm{U} \bar{\varepsilon}_{\mu}^{*} \bar{q}_{\nu}, \\
\left\langle\mathfrak{B}^{\prime}(k)\left|\bar{u} \sigma^{\mu \nu} \gamma_{5} c\right| \mathfrak{B}(k+\bar{q})\right\rangle \bar{\varepsilon}_{\mu}^{*} \bar{q}_{\nu} & =\overline{\mathrm{U}^{\prime}}\left\{\sigma^{\mu \nu} \gamma_{5} f_{1}^{(0)}-\epsilon^{\mu \nu \kappa \tau}\left[\gamma_{\kappa} \gamma_{\tau}(\not k+\not \bar{q})-\not k \gamma_{\kappa} \gamma_{\tau}\right] \frac{f_{2}^{(0)}}{4}\right\} \mathrm{U} \bar{\varepsilon}_{\mu}^{*} \bar{q}_{\nu} \\
& =f_{\mathfrak{B B}^{\prime}} \overline{\mathrm{U}^{\prime}} \sigma^{\mu \nu} \gamma_{5} \mathrm{U} \bar{\varepsilon}_{\mu}^{*} \bar{q}_{\nu},
\end{aligned}
$$

where $f_{1,2}^{(0)}$ stands for $f_{1,2}$ evaluated at $\bar{q}^{2}=0$ and

$$
f_{\mathfrak{B B}^{\prime}}=f_{1}^{(0)}+\frac{f_{2}^{(0)}}{2}\left(m_{\mathfrak{B}^{\prime}}-m_{\mathfrak{B}}\right)
$$

with $m_{\mathfrak{B}^{\prime \prime}}$ being the mass of $\mathfrak{B}^{(\prime)}$. These results lead to Eq. (13). Evidently, the same combination, $f_{\mathfrak{B B}^{\prime}}$, of form factors at $\bar{q}^{2}=0$ enters both Eqs. (A3) and (A4), in agreement with what was previously found [60,61] concerning the baryonic matrix elements of these types of tensor operators.

In the case where $\mathfrak{B}^{\prime}$ is replaced by a spin-3/2 baryon $\mathbb{B}^{\prime}$, we can write

$$
\begin{aligned}
\left\langle\mathbb{B}^{\prime}(k)\left|\bar{u} i \sigma^{\mu \nu} c\right| \mathfrak{B}(k+\bar{q})\right\rangle= & \left\{\overline{\mathbb{U}}^{[\mu} \gamma^{\nu]} g_{1}+\overline{\mathbb{U}}^{[\mu} k^{\nu]} g_{2}+\overline{\mathbb{U}}^{\eta} \bar{q}_{\eta}\left(i \sigma^{\mu \nu} g_{3}+\gamma^{[\mu} k^{\nu]} g_{4}\right)\right. \\
& \left.+\overline{\mathbb{U}}^{[\mu} \bar{q}^{\nu]} g_{5}+\overline{\mathbb{U}}^{\eta} \bar{q}_{\eta}\left(\gamma^{[\mu} \bar{q}^{\nu]} g_{6}+k^{[\mu} \bar{q}^{\nu]} g_{7}\right)\right\} \gamma_{5} \mathrm{U},
\end{aligned}
$$

where $k$ and $\mathbb{U}^{\mu}$ are, respectively, the momentum of $\mathbb{B}^{\prime}$ and its Rarita-Schwinger spinor, the latter satisfying the requirements $\gamma_{\mu} \mathbb{U}^{\mu}=k_{\mu} \mathbb{U}^{\mu}=0$, and $g_{1,2, \ldots, 7}$ represent form factors which depend on $\bar{q}^{2}$. It follows that

$$
\begin{aligned}
\left\langle\mathbb{B}^{\prime}(k)\left|\bar{u} i \sigma^{\mu \nu} c\right| \mathfrak{B}(k+\bar{q})\right\rangle \bar{\varepsilon}_{\mu}^{*} \bar{q}_{\nu}= & \overline{\mathbb{U}}\left[\mu \gamma^{\nu]} \bar{\varepsilon}_{\mu}^{*} \bar{q}_{\nu}\left[g_{1}^{(0)}+\frac{g_{2}^{(0)}}{2}\left(m_{\mathbb{B}^{\prime}}-m_{\mathfrak{B}}\right)\right] \gamma_{5} \mathrm{U}\right. \\
& +\overline{\mathbb{U}}^{\eta} \bar{q}_{\eta} \gamma^{\tau} \bar{\varepsilon}_{\tau}^{*} \bar{\phi}\left[\frac{g_{2}^{(0)}}{2}-g_{3}^{(0)}-\frac{g_{4}^{(0)}}{2}\left(m_{\mathbb{B}^{\prime}}+m_{\mathfrak{B}}\right)\right] \gamma_{5} \mathrm{U} \\
= & \overline{\mathbb{U}} \eta\left[\left(\bar{\varepsilon}_{\eta}^{*} \not \bar{q}-\gamma^{\tau} \bar{\varepsilon}_{\tau}^{*} \bar{q}_{\eta}\right) \mathcal{F}+\frac{\bar{q}_{\eta} \gamma^{\tau} \bar{\varepsilon}_{\tau}^{*} \not \tilde{\mathcal{F}} \tilde{\mathcal{F}}}{m_{\mathfrak{B}}}\right] \gamma_{5} \mathrm{U},
\end{aligned}
$$

where $g_{j}^{(0)}$ denotes the value of $g_{j}$ at $\bar{q}^{2}=0$ and 


$$
\mathcal{F}=g_{1}^{(0)}+\frac{g_{2}^{(0)}}{2}\left(m_{\mathbb{B}^{\prime}}-m_{\mathfrak{B}}\right), \quad \frac{\tilde{\mathcal{F}}}{m_{\mathfrak{B}}}=\frac{g_{2}^{(0)}}{2}-g_{3}^{(0)}-\frac{g_{4}^{(0)}}{2}\left(m_{\mathbb{B}^{\prime}}+m_{\mathfrak{B}}\right),
$$

with $m_{\mathbb{B}^{\prime}}$ being the mass of $\mathbb{B}^{\prime}$. Moreover,

$$
\begin{aligned}
\left\langle\mathbb{B}^{\prime}(k)\left|\bar{u} i \sigma^{\mu \nu} \gamma_{5} c\right| \mathfrak{B}(k+\bar{q})\right\rangle \bar{\varepsilon}_{\mu}^{*} \bar{q}_{\nu} & =\frac{\epsilon^{\mu \nu \kappa \tau}}{2}\left\langle\mathbb{B}^{\prime}(k)\left|\bar{u} \sigma_{\kappa \tau} c\right| \mathfrak{B}(k+\bar{q})\right\rangle \bar{\varepsilon}_{\mu}^{*} \bar{q}_{\nu} \\
& =\overline{\mathbb{U}}^{\eta}\left[\left(\gamma^{\tau} \bar{\varepsilon}_{\tau}^{*} \bar{q}_{\eta}-\bar{\varepsilon}_{\eta}^{*} \bar{q}\right) \mathcal{F}+\frac{\bar{q}_{\eta} \gamma^{\tau} \bar{\varepsilon}_{\tau}^{*} \not \overline{\mathcal{F}}}{m_{\mathfrak{B}}}\right] \mathrm{U} .
\end{aligned}
$$

From Eqs. (A7) and (A9), we get Eq. (20).

In the derivation of the $\mathfrak{B} \rightarrow \mathbb{B}^{\prime} \gamma$ rate, the absolute square of the decay amplitude needs to be summed over the initial and final particles' polarizations. The expression for the sum over the $\mathbb{B}^{\prime}$ polarizations is available from the literature (e.g., [62]). It is given here for completeness,

$$
\sum_{\varsigma=-3 / 2}^{3 / 2} \mathbb{U}_{\varsigma}^{\beta}(k) \bar{\cup}_{\varsigma}^{\eta}(k)=\left(\not k+m_{\mathbb{B}^{\prime}}\right)\left(\frac{\gamma_{\mu} \gamma_{\nu}}{3} \mathcal{G}^{\mu \beta}(k) \mathcal{G}^{\nu \eta}(k)-\mathcal{G}^{\beta \eta}(k)\right),
$$

where $\mathcal{G}^{\mu \nu}(k)=g^{\mu \nu}-k^{\mu} k^{\nu} / m_{\mathbb{B}^{\prime}}^{2}$.

[1] L. B. Okun, Limits of electrodynamics: Paraphotons?, Zh. Eksp. Teor. Fiz. 83, 892 (1982) [Sov. Phys. JETP 56, 502 (1982)], http://www.jetp.ac.ru/cgi-bin/e/index/e/56/3/p502? $\mathrm{a}=$ list.

[2] H. Georgi, P. H. Ginsparg, and S.L. Glashow, Photon oscillations and the cosmic background radiation, Nature (London) 306, 765 (1983).

[3] B. Holdom, Two U(1)'s and $\epsilon$ charge shifts, Phys. Lett. 166B, 196 (1986).

[4] R. Foot and X.G. He, Comment on $Z-Z^{\prime}$ mixing in extended gauge theories, Phys. Lett. B 267, 509 (1991).

[5] F. del Aguila, M. Masip, and M. Perez-Victoria, Physical parameters and renormalization of $U(1)^{a} \times U(1)^{b}$ models, Nucl. Phys. B456, 531 (1995).

[6] B. A. Dobrescu, Massless Gauge Bosons Other than the Photon, Phys. Rev. Lett. 94, 151802 (2005).

[7] E. Gabrielli, B. Mele, M. Raidal, and E. Venturini, FCNC decays of standard model fermions into a dark photon, Phys. Rev. D 94, 115013 (2016).

[8] S. Hoffmann, Paraphotons and axions: Similarities in stellar emission and detection, Phys. Lett. B 193, 117 (1987).

[9] D. Fargion, M. Khlopov, and C. A. Stephan, Cold dark matter by heavy double charged leptons?, Classical Quantum Gravity 23, 7305 (2006).

[10] L. Ackerman, M. R. Buckley, S. M. Carroll, and M. Kamionkowski, Dark matter and dark radiation, Phys. Rev. D 79, 023519 (2009).

[11] V. Barger, C. W. Chiang, W. Y. Keung, and D. Marfatia, Constraint on Parity-Violating Muonic Forces, Phys. Rev. Lett. 108, 081802 (2012).

[12] C. W. Chiang and P. Y. Tseng, Probing a dark photon using rare leptonic kaon and pion decays, Phys. Lett. B 767, 289 (2017).
[13] M. He, X. G. He, C. K. Huang, and G. Li, Search for a heavy dark photon at future $e^{+} e^{-}$colliders, J. High Energy Phys. 03 (2018) 139.

[14] J.X. Pan, M. He, X. G. He, and G. Li, Scrutinizing a massless dark photon: Basis independence, Nucl. Phys. B953, 114968 (2020).

[15] M. Fabbrichesi, E. Gabrielli, and B. Mele, Hunting Down Massless Dark Photons in Kaon Physics, Phys. Rev. Lett. 119, 031801 (2017).

[16] J. Y. Su and J. Tandean, Kaon decays shedding light on massless dark photons, Eur. Phys. J. C 80, 824 (2020).

[17] J. Y. Su and J. Tandean, Searching for dark photons in hyperon decays, Phys. Rev. D 101, 035044 (2020).

[18] M. Pospelov, Secluded U(1) below the weak scale, Phys. Rev. D 80, 095002 (2009).

[19] J. Jaeckel and A. Ringwald, The low-energy frontier of particle physics, Annu. Rev. Nucl. Part. Sci. 60, 405 (2010).

[20] R. Essig et al., Working Group Report: New light weakly coupled particles, arXiv:1311.0029.

[21] J. Alexander et al., Dark sectors 2016 workshop: Community report, arXiv:1608.08632.

[22] M. Fabbrichesi, E. Gabrielli, and G. Lanfranchi, The dark photon, arXiv:2005.01515.

[23] J. R. Batley et al. (NA48/2 Collaboration), Search for the dark photon in $\pi^{0}$ decays, Phys. Lett. B 746, 178 (2015).

[24] M. Ablikim et al. (BESIII Collaboration), Dark photon search in the mass range between 1.5 and $3.4 \mathrm{GeV} / c^{2}$, Phys. Lett. B 774, 252 (2017).

[25] R. Aaij et al. (LHCb Collaboration), Search for Dark Photons Produced in $13 \mathrm{TeV} p p$ Collisions, Phys. Rev. Lett. 120, 061801 (2018). 
[26] A. Anastasi et al. (KLOE-2 Collaboration), Combined limit on the production of a light gauge boson decaying into $\mu^{+} \mu^{-}$ and $\pi^{+} \pi^{-}$, Phys. Lett. B 784, 336 (2018).

[27] M. Ablikim et al. (BESIII Collaboration), Measurement of $\mathcal{B}\left(J / \psi \rightarrow \eta^{\prime} e^{+} e^{-}\right)$and search for a dark photon, Phys. Rev. D 99, 012013 (2019).

[28] E. C. Gil et al. (NA62 Collaboration), Search for production of an invisible dark photon in $\pi^{0}$ decays, J. High Energy Phys. 05 (2019) 182.

[29] D. Banerjee et al., Dark Matter Search in Missing Energy Events with NA64, Phys. Rev. Lett. 123, 121801 (2019).

[30] M. Tanabashi et al. (Particle Data Group), Review of particle physics, Phys. Rev. D 98, 030001 (2018).

[31] G. Buchalla, A. J. Buras, and M. E. Lautenbacher, Weak decays beyond leading logarithms, Rev. Mod. Phys. 68, 1125 (1996).

[32] G. Burdman, E. Golowich, J. L. Hewett, and S. Pakvasa, Rare charm decays in the standard model and beyond, Phys. Rev. D 66, 014009 (2002).

[33] E. C. Gil et al. (NA62 Collaboration), The beam and detector of the NA62 experiment at CERN, J. Instrum. 12, P05025 (2017).

[34] J. K. Ahn et al. (KOTO Collaboration), Search for the $K_{L} \rightarrow$ $\pi^{0} \nu \bar{\nu}$ and $K_{L} \rightarrow \pi^{0} X^{0}$ Decays at the J-PARC KOTO Experiment, Phys. Rev. Lett. 122, 021802 (2019).

[35] H. B. Li, Prospects for rare and forbidden hyperon decays at BESIII, Front. Phys. (Beijing) 12, 121301 (2017), https:// doi.org/10.1007/s11467-017-0691-9.

[36] M. Ablikim et al., Future physics programme of BESIII, Chin. Phys. C 44, 040001 (2020).

[37] D. M. Asner et al., Physics at BES-III, Int. J. Mod. Phys. A 24, 499 (2009).

[38] E. Kou et al. (Belle-II Collaboration), The Belle II physics book, Prog. Theor. Exp. Phys. 2019, 123 C01 (2019); Erratum, Prog. Theor. Exp. Phys. 2020, 029201 (2020).

[39] Y. T. Lai et al. (Belle Collaboration), Search for $D^{0}$ decays to invisible final states at Belle, Phys. Rev. D 95, 011102 (2017).

[40] Q. Luo and D. Xu, Progress on preliminary conceptual study of HIEPA, a Super Tau-Charm factory in China, in Proceedings of the IPAC2018, Vancouver, British Columbia, Canada (JACoW Publishing, Geneva, Switzerland, 2018), http://accelconf.web.cern.ch/ipac2018/doi/JACoWIPAC2018-MOPML013.html.

[41] A. Y. Barnyakov (Super Charm-Tau Factory Collaboration), The project of the Super Charm-Tau factory in Novosibirsk, J. Phys. Conf. Ser. 1561, 012004 (2020).

[42] J. B. G. da Costa et al. (CEPC Study Group), CEPC conceptual design report: Volume 2-physics \& detector, arXiv:1811.10545.

[43] N. Isgur and M. B. Wise, Relationship between form-factors in semileptonic $\bar{B}$ and $D$ decays and exclusive rare $\bar{B}$ meson decays, Phys. Rev. D 42, 2388 (1990).

[44] D. Melikhov and B. Stech, Weak form-factors for heavy meson decays: An update, Phys. Rev. D 62, 014006 (2000).

[45] Y.L. Wu, M. Zhong, and Y.B. Zuo, $B(s), D(s) \rightarrow$ $\pi, K, \eta, \rho, K^{*}, \omega, \phi$ transition form factors and decay rates with extraction of the CKM parameters $|V(u b)|,|V(c s)|$, $|V(c d)|$, Int. J. Mod. Phys. A 21, 6125 (2006).

[46] C. Q. Geng, C. C. Lih, and W. M. Zhang, Study of radiative leptonic $D$ meson decays, Mod. Phys. Lett. A 15, 2087 (2000).

[47] D. E. Hazard and A. A. Petrov, Radiative lepton flavor violating $B, D$, and $K$ decays, Phys. Rev. D 98, 015027 (2018).

[48] S. Meinel, $\Lambda_{c} \rightarrow N$ form factors from lattice QCD and phenomenology of $\Lambda_{c} \rightarrow n \ell^{+} \nu_{\ell}$ and $\Lambda_{c} \rightarrow p \mu^{+} \mu^{-}$decays, Phys. Rev. D 97, 034511 (2018).

[49] R. N. Faustov and V. O. Galkin, Rare $\Lambda_{c} \rightarrow p \ell^{+} \ell^{-}$decay in the relativistic quark model, Eur. Phys. J. C 78, 527 (2018).

[50] K. Azizi, Y. Sarac, and H. Sundu, Light cone QCD sum rules study of the semileptonic heavy $\Xi_{Q}$ and $\Xi_{Q}^{\prime}$ transitions to $\Xi$ and $\Sigma$ baryons, Eur. Phys. J. A 48, 2 (2012).

[51] A. Cerri et al., Report from Working Group 4: Opportunities in flavour physics at the HL-LHC and HE-LHC, CERN Yellow Rep. Monogr. 7, 867 (2019), https://e-publishing .cern.ch/index.php/CYRM/article/view/954.

[52] R. Aaij et al. (LHCb Collaboration), Observation of the Doubly Charmed Baryon $\Xi_{c c}^{++}$, Phys. Rev. Lett. 119, 112001 (2017).

[53] X.H. Hu, R.H. Li, and Z.P. Xing, A comprehensive analysis of weak transition form factors for doubly heavy baryons in the light front approach, Eur. Phys. J. C 80, 320 (2020).

[54] G. Hiller, M. Knecht, F. Legger, and T. Schietinger, Photon polarization from helicity suppression in radiative decays of polarized $\Lambda_{b}$ to spin-3/2 baryons, Phys. Lett. B 649, 152 (2007).

[55] T. M. Aliev and M. Savci, Analysis of the semileptonic $B_{c} \rightarrow B_{u}^{*} \ell^{+} \ell^{-}$decay from QCD sum rules, Eur. Phys. J. C 47, 413 (2006).

[56] S. Fajfer, S. Prelovsek, and P. Singer, FCNC transitions $c \rightarrow u \gamma$ and $s \rightarrow d \gamma$ in $B_{c} \rightarrow B_{u}^{*} \gamma$ and $B_{s} \rightarrow B_{d}^{*} \gamma$ decays, Phys. Rev. D 59, 114003 (1999); Erratum, Phys. Rev. D64, 099903 (2001).

[57] N. Isgur, D. Scora, B. Grinstein, and M. B. Wise, Semileptonic $B$ and $D$ decays in the quark model, Phys. Rev. D 39, 799 (1989).

[58] C. H. Chen and C.Q. Geng, Baryonic rare decays of $\Lambda_{b} \rightarrow \Lambda l^{+} l^{-}$, Phys. Rev. D 64, 074001 (2001).

[59] Y. M. Wang, Y. Li, and C. D. Lu, Rare decays of $\Lambda_{b} \rightarrow$ $\Lambda+\gamma$ and $\Lambda_{b} \rightarrow \Lambda+l^{+} l^{-}$in the light-cone sum rules, Eur. Phys. J. C 59, 861 (2009).

[60] G. Hiller and A. Kagan, Probing for new physics in polarized $\Lambda_{b}$ decays at the $Z$, Phys. Rev. D 65, 074038 (2002).

[61] T. Gutsche, M. A. Ivanov, J. G. Korner, V. E. Lyubovitskij, and P. Santorelli, Rare baryon decays $\Lambda_{b} \rightarrow \Lambda \ell^{+} \ell^{-}(\ell=$ $e, \mu, \tau)$ and $\Lambda_{b} \rightarrow \Lambda \gamma$ : Differential and total rates, leptonand hadron-side forward-backward asymmetries, Phys. Rev. D 87, 074031 (2013).

[62] N. D. Christensen, P. de Aquino, N. Deutschmann, C. Duhr, B. Fuks, C. Garcia-Cely, O. Mattelaer, K. Mawatari, B. Oexl, and Y. Takaesu, Simulating spin- $\frac{3}{2}$ particles at colliders, Eur. Phys. J. C 73, 2580 (2013). 